\title{
Literacy-sensitive Intervention for COPD Patients
}

\author{
Madeleine Shapiro, BA, Valerie G. Press, MD, MPH, and Vineet M. Arora, MD, MAPP
}

University of Chicago, Chicago, IL, USA.

J Gen Intern Med 27(9): 1103

DOI: $10.1007 / \mathrm{s} 11606-012-2085-6$

(c) Society of General Internal Medicine 2012

To the Editors:-We commend Kiser et al. for their work addressing the impact of a literacy-sensitive, self-management intervention on improving inhaler technique for patients with chronic obstructive pulmonary disease (COPD). ${ }^{1}$ Given the high prevalence of inhaler misuse among patients with $\mathrm{COPD},{ }^{2}$ there is a great need for targeted approaches such as those utilized in this study.

We applaud their use of verbal administration for the majority of the study. However, because the commonly used Short Test of Functional Health Literacy in Adults (S-TOFHLA) is a written tool, we wonder what role poor vision might have played in classifying participants' health literacy. In studies at our institution, we found that among hospitalized patients one-third of participants had poor vision (worse than 20/50 vision in both eyes), $56 \%$ of whom were found to have poor health literacy using a verbal tool. ${ }^{3}$ This raised concern that poor vision is an additional, unaddressed risk factor for hospitalized patients with COPD.

This is salient because research has shown that poor vision can be a separate barrier to self-management, such as the ability to open medication containers, ${ }^{4}$ and among hospitalized patients, appropriate inhaler technique. ${ }^{2}$ Given these findings, it is important to understand the role of vision for self-management strategies, with respect to assessing health literacy level and/or whether poor vision compounds health risks associated with low health literacy.
Lastly, the average age in Kiser's study was 63 years old. Because older adults are at increased risk for low health literacy, ${ }^{5}$ it may be especially important to understand the association between health literacy and vision for older patients. We have found that patients aged 65 years or older are at a dual risk for low health literacy and poor vision, compared to their younger counterparts. ${ }^{3}$ With the rising numbers of older Americans, it is becoming even more important to differentiate between inhaler misuse due to poor vision and misuse due to low health literacy, as interventions may differ. Future work should attempt to achieve greater clarity between these risk factors.

Corresponding Author: Madeleine Shapiro, BA; University of Chicago, 5841 S. Maryland Ave, Chicago, IL 60637, USA (e-mail: mishapiro@uchicago.edu).

\section{REFERENCES}

1. Kiser K, Jonas D, Warner Z, Scanlon K, Bryant Shilliday B, DeWalt D. A randomized controlled trial of a literacy-sensitive self-management intervention for chronic obstructive pulmonary disease patients. J Gen Intern Med. 2012;27(2):190-5.

2. Press VG, Arora VM, Shah LM, et al. Misuse of respiratory inhalers in hospitalized patients with asthma or COPD. J Gen Intern Med. 2011;26 (6):635-42.

3. Press, VG, Shapiro, MI, Mayo, AM, Meltzer, Do, Arora VM. More than meets the eye: measuring the dual risks of low health literacy and poor vision in hospitalized patients. Abstract. J Hosp Med. 2012;7(2):S47.

4. Beckman A, Bernstein C, Parker MG, Thorslund M, Fastbom J. The difficulty of opening medicines containers in old age: a population based study. Pharm World Sci. 2005;27:393-8.

5. Baker DW, Gazmararian JA, Sudano J, Patterson M. The association between age and health literacy among elderly persons. J Gerontol B Psychol Sci Soc Sci. 2000;55(6):S368-S374. 